\title{
Qualitative Analysis of Indoor and Outdoor Airborne Fungi in Cowshed
}

\author{
R. Pavan and K. Manjunath \\ Department of Microbiology and Biotechnology, Bangalore University, Bangalore 560 056, India \\ Correspondence should be addressed to R. Pavan; pavanrgowda@rediffmail.com
}

Received 10 June 2014; Revised 28 August 2014; Accepted 28 August 2014; Published 16 September 2014

Academic Editor: Simona Nardoni

Copyright ( 2014 R. Pavan and K. Manjunath. This is an open access article distributed under the Creative Commons Attribution License, which permits unrestricted use, distribution, and reproduction in any medium, provided the original work is properly cited.

\begin{abstract}
Air pollution is one of the most serious problems to human health. Fungi are the causal agents for different diseases in animals, plants, and human beings. Otomycosis, chronic bronchitis, emphysema, asthma, allergy, and systemic mycosis are among the fungal diseases caused. The present study was conducted to analyze the monthly incidence of airborne fungi, seasonal variation, and influence of meteorological parameters in indoor and outdoor fungi of cowshed at Hesaraghatta village, Bangalore. An aeromycological survey of indoor and outdoor area of cowshed at Hesaraghatta village in Bangalore city was carried out using the Andersen two-stage sampler onto a petri dish containing malt extract agar from January 2011 to December 2011. Altogether, 29 species belonging to 13 genera from indoor and 26 species belonging to 12 genera were recorded from outdoor environment of the cowshed; the dominant fungal species identified were Cladosporium sp., Aspergillus sp., and Alternaria alternata. Seasonal occurrence of fungal spores in both indoor and outdoor of the cowshed revealed that maximum spores were recorded in summer season followed by winter and rainy season.
\end{abstract}

\section{Introduction}

Airborne particles are present throughout the environment. Despite the fact that atmospheric air does not favour growth of microorganisms due to lack of nutrients, the microorganisms are present in aerosol form, suspended in the air. The basic sources of microbes are soil, water, animals, and humans and they originate in many different forms and affect visibility, climate, human health, and the quality of life [1]. Airborne microbial quantity and quality vary with time of day, year, and location [2].

Fungi are common in indoor and outdoor environment. Nearly $10 \%$ of people worldwide have fungal allergy [3]. Numerous studies have shown that exposure to fungi may be associated with acute toxic effects, allergies, and asthma [4]. Researchers believe that more than 80 genera of fungi are associated with symptoms of respiratory tract allergies [5]. Over 100 species of fungi are involved with serious human and animal infections, whereas many other species cause serious plant diseases [6]. Many fungal spores are involved in respiratory allergies and different kinds of infections [7].
Fungal aerosols produced in animal rearing houses may threaten caretakers and external environment. Respiratory infection or damage may occur in caretakers as well as in animal rearing houses with prolonged exposure to the environment at high microorganism levels [8]. Microbial aerosols of high levels are also associated with allergy and asthma in caretakers [9]. Airborne fungi have much attention from medical researchers as well as environmentalists [10]. At present, there are numerous studies highlighting both outdoor and indoor environments, especially in buildings frequented by a large number of people, who may be exposed to this type of aeroallergen [11], and many authors indicated that the dominant fungi were and in the atmosphere and their concentration differed from place to place, because of local environmental variables, fungal substrates, and human activities.

Many studies have investigated the concentrations of airborne fungal spores from cowshed places and a relatively high frequency of Aspergillus sp., Cladosporium sp., Curvularia sp., Penicillium sp., Alternaria sp., Fusarium sp., Helminthosporium sp., and Trichoderma species was recorded 
$[12,13]$. The type of breeding, the method of feed distribution, and airflow velocity in animal rearing houses were suggested as the agents significantly modifying the level of fungi [14].

Airborne fungal spore is related to multiple aerodynamic factors, such as spore size, shape, weight, and electrostatic properties of their walls $[15,16]$. Geographical location, climate, and meteorological factors (temperature, wind speed, relative humidity, and rainfall) are responsible for indoor and outdoor types and levels of fungal spores behavior and dispersion in the atmosphere $[17,18]$. The present study was conducted to analyze the monthly incidence of airborne fungi, seasonal variation, and influence of meteorological parameters in indoor and outdoor fungi of cowshed at Hesaraghatta village, Bangalore.

\section{Materials and Methods}

2.1. Sampling Site. The cowshed selected for this investigation was situated at Hesaraghatta village in Bangalore, India.

2.2. Sampling Time. Indoor and outdoor air samples of cowshed were collected at monthly intervals over a period from January 2011 to December 2011.

2.3. Collection of Samples. Andersen two-stage sampler was placed in the center of the cowshed [19], 1.5 meter above the ground level. Malt extract agar was used as sampling medium. Airflow was $28.3 \mathrm{~L} / \mathrm{min}$ during the sampling and the sampling time is limited to 5 minutes.

2.4. Treatment of Samples. The air sampled plates were incubated for 5 to 7 days at room temperature and the identification was based on the detailed study of all the microscopic morphological characters of the fungi and the macroscopic features of their colonies. The results for each stage of the sampler were expressed as colony forming units per cubic meter of air $\left(\mathrm{CFU} / \mathrm{m}^{3}\right)$ and total concentration was obtained by adding the $\mathrm{CFU} / \mathrm{m}^{3}$ from each plate. Identification of fungal colonies was based on morphological characteristics and microscopic observations with the help of Agharkar Research Institute, Pune (India).

2.5. Statistical Analysis. Statistical analysis was carried out using SPSS windows Microsoft version 16, 2007. Comparison between indoor and outdoor airborne fungi, Pearson's correlation between meteorological variables tests, one-way ANOVA for CFU, and two-way ANOVA for CFU and season of the cowshed were performed with significance at the 0.05 level 2-tailed.

\section{Results}

During the study period, a total number of $15419.04 \mathrm{CFU} / \mathrm{m}^{3}$ was isolated from both indoor and outdoor environment of cowshed, of which indoor $7808.36 \mathrm{CFU} / \mathrm{m}^{3}$ and outdoor $7610.68 \mathrm{CFU} / \mathrm{m}^{3}$ of cowshed contributed (Tables 1 and 2). Altogether 29 species belonging to 13 genera were isolated from indoor and outdoor 26 species belonging to 12 genera isolated, in addition to unidentified fungi.

Among the total number of isolated fungal species from indoor Aspergillus (27.36\%) was represented by 6 species, namely, A. flavus, A. niger, A. oryzae, A. ochraceus, $A$. fumigatus, and A. terreus, followed by 3 species of Cladosporium (18.2\%), namely, C. cladosporioides, C. herbarium, and C. acremonium; 3 species of Fusarium (13.9\%), namely, F. oxysporum, F. moniliforme, and F. solani; 3 species of Penicillium (13.14\%), namely, $P$. versicolor, $P$. citrinum, and $P$. nigricans; and 1 species of Curvularia (4.07\%), namely, C. lunata, along with Acremonium sp. (2.07\%), Alternaria alternata (5.51\%), Ascomycetes sp. (0.85\%), Mucor sp. (2.66\%), Neurospora sp. (0.72\%), Rhizopus sp. (4.06\%), Scopulariopsis sp. (0.4\%), and Trichoderma sp. (3.2\%) which are presented in Table 1. When these findings were compared with outdoor Aspergillus (22.19\%) was represented by 5 species, namely, A. flavus, A. niger, A. oryzae, A. fumigatus, and A. terreus, followed by 3 species of Cladosporium (15.89\%), namely, $C$. cladosporioides, C. herbarium, and C. acremonium; 3 species of Fusarium (12.59\%), namely, F. oxysporum, F. moniliforme, and F. solani; 2 species of Penicillium (10.14\%), namely, $P$. versicolor and P. citrinum; and 1 species of Curvularia (4.07\%), namely, C. lunata, along with Acremonium sp. (4.08\%), Alternaria alternata (10.85\%), Ascomycetes sp. (4.22\%), Mucor sp. (4.99\%), Rhizopus sp. (3.1\%), Scopulariopsis sp. (0.88\%), and Trichoderma sp. (4.35\%) which are presented in Table 2. It was observed that all the fungal genera and species remain the same, with variation in their percentage occurrence, but few of the fungal species like A. ochraceus, Neurospora sp., and $P$. nigricans were found in the indoor environment of the cowshed.

Based on comparative analysis, the dominant fungal species in indoor of cowshed were Cladosporium $(8.13 \%)$, Aspergillus (6.41\%), and Aspergillus niger (6.1\%) but Scopulariopsis (0.4\%) and Aspergillus ochraceus (0.63\%) were the least recorded (Table 1) but in outdoor the dominant fungal species were Alternaria alternata (10.85\%) and Aspergillus (10.71\%) while Scopulariopsis (0.88\%) and Aspergillus terreus $(0.97 \%)$ were the least recorded (Table 2$)$. Monthly variation of cowshed total fungal spores in indoor showed maximum spore distribution in May $\left(780.13 \mathrm{CFU} / \mathrm{m}^{3}\right)$ followed by February $\left(773.07 \mathrm{CFU} / \mathrm{m}^{3}\right)$ and January $\left(755.42 \mathrm{CFU} / \mathrm{m}^{3}\right)$ and outdoor showed the highest distribution during March $\left(790.72 \mathrm{CFU} / \mathrm{m}^{3}\right)$ followed by June $\left(783.66 \mathrm{CFU} / \mathrm{m}^{3}\right)$ and January $\left(716.59 \mathrm{CFU} / \mathrm{m}^{3}\right)$ when compared to other months of the year.

Seasonal occurrence of fungal spores in both indoor and outdoor air of cowshed (Table 3 ) revealed almost similar percentages of fungal spores in the respective seasons studied. During summer season, maximum spores were recorded $2742.81 \mathrm{CFU} / \mathrm{m}^{3}$ followed by winter $2643.97 \mathrm{CFU} / \mathrm{m}^{3}$ and rainy $2421.58 \mathrm{CFU} / \mathrm{m}^{3}$ in indoor. For outdoor, summer season contributed maximum $2756.93 \mathrm{CFU} / \mathrm{m}^{3}$, followed by winter $2548.66 \mathrm{CFU} / \mathrm{m}^{3}$ and rainy $2305.09 \mathrm{CFU} / \mathrm{m}^{3}$ season. Over all, maximum spore formation due to the abundance of Aspergillus flavus (218.86 CFU/m $\mathrm{m}^{3}$ ) was observed during summer season, followed by winter season with maximum 
TABLE 1: Monthly occurrence of fungal spores $\left(\mathrm{CFU} / \mathrm{m}^{3}\right)$ recorded from January 2011 to December 2011 in the indoor air of cowshed.

\begin{tabular}{|c|c|c|c|c|c|c|c|c|c|c|c|c|c|c|c|}
\hline Sl. n & Genera and species & Jan. & Feb. & Mar. & Apr. & May & Jun. & Jul. & Aug. & Sep. & Oct. & Nov. & Dec. & Total & $\%$ \\
\hline 1 & Acremonium sp. & 0 & 0 & 7.06 & 3.53 & 52.95 & 0 & 14.12 & 31.77 & 0 & 49.42 & 0 & 3.53 & 162.38 & 2.07 \\
\hline 2 & Alternaria alternata & 45.89 & 63.54 & 35.3 & 14.12 & 31.77 & 35.3 & 35.3 & 21.18 & 42.36 & 21.18 & 42.36 & 42.36 & 430.66 & 5.51 \\
\hline 3 & Ascomycetes sp. & 0 & 0 & 10.59 & 3.53 & 3.53 & 3.53 & 3.53 & 14.12 & 7.06 & 21.18 & 0 & 0 & 67.07 & 0.85 \\
\hline 4 & Aspergillus spp. & 56.48 & 91.78 & 49.42 & 24.71 & 28.24 & 35.3 & 21.18 & 10.59 & 45.89 & 67.07 & 35.3 & 35.3 & 501.26 & 6.41 \\
\hline 5 & & 38.83 & 98.84 & 52.95 & 45.89 & 98.84 & 21.18 & 21.18 & 31.77 & 14.12 & 21.18 & 10.59 & 7.06 & 462.43 & 5.92 \\
\hline 6 & & 77.66 & 63.54 & 49.42 & 9.42 & 24.71 & 56.48 & 28.24 & 56.48 & 35.3 & 0 & 0 & 35.3 & 476.55 & 6.1 \\
\hline & A. oryzae & 7.06 & 3.53 & 21.18 & 38.83 & 28.24 & 38.83 & 17.65 & 42.36 & 28.24 & 21.18 & 28.24 & 52.95 & 328.29 & 4.2 \\
\hline 8 & A. ochraceus & 0 & 0 & 3.53 & 10.59 & 0 & 3.53 & 3.53 & 0 & 7.06 & 7.06 & 7.06 & 7.06 & 49.42 & 0.63 \\
\hline 9 & A. fumigatus & 0 & 0 & 14.12 & 21.18 & 0 & 0 & 10.59 & 24.71 & 17.65 & 10.59 & 0 & 0 & 98.84 & 1.26 \\
\hline 10 & & 24.71 & 17.65 & 14.12 & 3.53 & 3.53 & 10.59 & 31.77 & 7.06 & 10.59 & 31.77 & 28.24 & 38.83 & 222.39 & 2.84 \\
\hline 11 & & 83 & 17.65 & 14.12 & 21.18 & 7.06 & 0 & 0 & 17.65 & 21.18 & 0 & 45.89 & 5.3 & .86 & 2.8 \\
\hline 12 & & 10.59 & 0 & & 3.53 & 0 & 10.59 & 21.18 & 0 & 0 & 49.42 & 28.24 & & & 2.16 \\
\hline 13 & Cladosporium spp. & 63.54 & 56.48 & 98.84 & 81.19 & 77.66 & 60.01 & 28.24 & 28.24 & 49.42 & 35.3 & 21.18 & 35.3 & 635.4 & 8.13 \\
\hline 14 & C. cladosporioides & 35.3 & 49.42 & 21.18 & 7.06 & 35.3 & 49.42 & 28.24 & 56.48 & 49.42 & 7.06 & 14.12 & 42.36 & 395.36 & 5.06 \\
\hline 15 & C. herbarum & 0 & 0 & 49.42 & 28.24 & 35.3 & 42.36 & 31.77 & 10.59 & 42.36 & 17.65 & 0 & 0 & 257.69 & 3.3 \\
\hline 16 & & 7.06 & 21.18 & 0 & 706 & 14.12 & 14.12 & 21.18 & 35.3 & 7.06 & 0 & 0 & 7.06 & 34.14 & 1.71 \\
\hline 17 & & 77.66 & 28.24 & 60.01 & 83 & 14.12 & 17.65 & 12 & 14.12 & 14.12 & 45.89 & 3.53 & 10.59 & 88 & 4.33 \\
\hline 18 & & 38.83 & 10.59 & 42.36 & 28.24 & 7.06 & 0 & 14.12 & 7.06 & 17.65 & 7.06 & 21.18 & 12 & 27 & 2.66 \\
\hline 19 & & 0 & 35.3 & 21.18 & 42.36 & 91.78 & 0 & 21.18 & 7.06 & 0 & 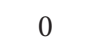 & 17.65 & 35.3 & 271.81 & 3.48 \\
\hline 20 & F. solani & 14.12 & 17.65 & 3.53 & 10.59 & 21.18 & 35.3 & 56.48 & 42.36 & 3.53 & 14.12 & 35.3 & 14.12 & 268.28 & 3.43 \\
\hline 21 & Mucor sp. & 38.83 & 14.12 & 14.12 & 21.18 & 0 & 21.18 & 17.65 & 14.12 & 14.12 & 0 & 14.12 & 38.83 & 208.27 & 2.66 \\
\hline 22 & Neurospora sp. & 0 & 0 & 7.06 & 14.12 & 10.59 & 0 & 0 & 3.53 & 14.12 & 7.06 & 0 & 0 & 56.48 & 0.72 \\
\hline 23 & Rhizopus sp. & 21.18 & 35.3 & 14.12 & 45.89 & 42.36 & 3.53 & 10.59 & 28.24 & 42.36 & 24.71 & 14.12 & 35.3 & 317.7 & 4.06 \\
\hline 24 & & 49.42 & 17.65 & 35.3 & 24.71 & 42.36 & 17.65 & 21.18 & 42.36 & 3.53 & 21.18 & 28.24 & 31.77 & 335.35 & 4.29 \\
\hline 25 & & 31.77 & 31.77 & 14.12 & 21.18 & 14.12 & 14.12 & 42.36 & 3.53 & 35.3 & 7.06 & 24.71 & 17.65 & 257.69 & 3.3 \\
\hline 26 & P. citrinum & 14.12 & 21.18 & 21.18 & 38.83 & 21.18 & 21.18 & 7.06 & 7.06 & 35.3 & 14.12 & 35.3 & 14.12 & 250.63 & 3.2 \\
\hline 27 & P. nigricans & 0 & 3.53 & 7.06 & 14.12 & 14.12 & 31.77 & 38.83 & 38.83 & 24.71 & 10.59 & 0 & 0 & 183.56 & 2.35 \\
\hline 28 & Scopulariopsis sp. & 7.06 & 3.53 & 0 & 0 & 0 & 0 & 7.06 & 7.06 & 3.53 & 3.53 & 0 & 0 & 31.77 & 0.4 \\
\hline 29 & Trichoderma sp. & 14.12 & 35.3 & 7.06 & 14.12 & 24.71 & 24.71 & 45.89 & 7.06 & 7.06 & 7.06 & 14.12 & 49.42 & 250.63 & 3.2 \\
\hline 30 & Unidentified & 42.36 & 35.3 & 10.59 & 0 & 35.3 & 7.06 & 31.77 & 17.65 & 7.06 & 24.71 & 7.06 & 0 & 218.86 & 2.8 \\
\hline & otal & 5.42 & 3.07 & 709.53 & 7.76 & 780.13 & 575.39 & 645.99 & 628.34 & 00.1 & 547.15 & 476.5 & 38.93 & 7808.36 & 100 \\
\hline
\end{tabular}

occurrence of Aspergillus spp. $\left(218.86 \mathrm{CFU} / \mathrm{m}^{3}\right)$ and in rainy season Cladosporium spp. $\left(141.20 \mathrm{CFU} / \mathrm{m}^{3}\right)$ were recorded.

Distribution of CFU varied with meteorological factors (Figure 1); at higher temperature of more than $32^{\circ} \mathrm{C}$, the total CFU's distribution was slightly more during March $709.53 \mathrm{CFU} / \mathrm{m}^{3}$ reached maximum in their CFU's, when the temperature was less than $26^{\circ} \mathrm{C}$, the CFU's reduced between November $476.55 \mathrm{CFU} / \mathrm{m}^{3}$. The high relative humidity from June to September (94\%) reduced the distribution of organisms $\left(2449.82 \mathrm{CFU} / \mathrm{m}^{3}\right)$. The $\mathrm{CFU}$ gradually increased to $2915.78 \mathrm{CFU} / \mathrm{m}^{3}$ with less relative humidity (85\%) between the months of January and April. In the present study, the wind speed showed less impact on the distribution of $\mathrm{CFU}$ with less variation in their CFU. Almost during the months from May to October with wind speed of $9 \mathrm{~km} / \mathrm{h}$ or higher, the distribution of organisms seems to be less $\left(3777.10 \mathrm{CFU} / \mathrm{m}^{3}\right)$, and at less wind speed from the months of November to April $(4.5 \mathrm{~km} / \mathrm{h})$ the organism number was more with $4031.26 \mathrm{CFU} / \mathrm{m}^{3}$. During the maximum rainfall in the month of August $(253.2 \mathrm{~mm})$ distribution of organism was $628.34 \mathrm{CFU} / \mathrm{m}^{3}$, but during January $755.42 \mathrm{CFU} / \mathrm{m}^{3}$ and March $709.53 \mathrm{CFU} / \mathrm{m}^{3}$ though there was no rainfall, the total organism distribution became higher during these periods.

The correlation of coefficient between meteorological parameters like temperature, relative humidity, wind speed, and rainfall with cowshed fungal CFU collected during the sampling time showed significance at different concentration levels (Table 4). Comparisons of Pearson's correlation between meteorological variables of the cowshed CFU and relative humidity are significantly negatively correlated (Table 5). One-way ANOVA for CFU of the cowshed between variations and within variations was not statistically 
TABLE 2: Monthly occurrence of fungal spores $\left(\mathrm{CFU} / \mathrm{m}^{3}\right)$ recorded from January 2011 to December 2011 in the outdoor air of cowshed.

\begin{tabular}{|c|c|c|c|c|c|c|c|c|c|c|c|c|c|c|c|}
\hline Sl. $n$ & Genera and species & Jan. & Feb. & Mar. & Apr. & May & Jun. & Jul. & Aug. & Sep. & Oct. & Nov. & Dec. & Total & $\%$ \\
\hline & Acremonium sp. & 0 & 0 & 24.71 & 21.18 & 35.3 & 31.77 & 24.71 & 2.95 & 31.77 & 8.25 & 0 & 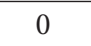 & 10.64 & 4.08 \\
\hline & Alternaria alternata & 7.66 & 8.25 & 52.95 & 35.3 & 95.31 & 112.96 & 91.78 & 45.89 & 60.01 & 74.13 & 70.6 & 21.18 & 826.02 & 10.85 \\
\hline & Ascomycetes sp. & 0 & 0 & 42.36 & 35.3 & 21.18 & 45.89 & 38.83 & 49.42 & 31.77 & 56.48 & 0 & 0 & 21.23 & 4.22 \\
\hline 4 & Aspergillus spp. & 12.9 & 95.31 & 74.13 & 56.48 & 49.42 & 70.6 & 38.83 & 31.77 & 35.3 & 74.13 & 102.37 & 74.13 & 15.43 & 10.71 \\
\hline 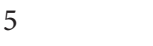 & A. flavus & 10.59 & 35.3 & 21.18 & 24.71 & 24.71 & 38.83 & 0 & 0 & 0 & 0 & 31.77 & 14.12 & 201.21 & 2.64 \\
\hline 6 & A. niger & 0 & 0 & 10.59 & 14.12 & 0 & 10.59 & 28.24 & 24.71 & 7.06 & 14.12 & 0 & 0 & 109.43 & 1.43 \\
\hline 7 & A. oryzae & 14.12 & 28.24 & 7.06 & 14.12 & 24.71 & 7.06 & 10.59 & 10.59 & 7.06 & 17.65 & 42.36 & 35.3 & 218.86 & 2.87 \\
\hline 8 & A. fumigatus & 21.18 & 24.71 & 28.24 & 49.42 & 7.06 & 7.06 & 17.65 & 28.24 & 24.71 & 24.71 & 14.12 & 24.71 & 271.81 & 3.57 \\
\hline 9 & & 3.53 & 0 & 14.12 & 24.71 & 0 & 7.06 & 3.53 & 0 & O & 0 & 3.53 & 17.65 & 74.13 & 0.97 \\
\hline 10 & & 14.12 & 7.06 & 21.18 & 7.06 & 3.53 & 14.12 & 0 & 24.71 & 35.3 & 35.3 & 14.12 & 3.53 & 03 & 2.36 \\
\hline 11 & & 0 & 0 & 0 & 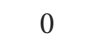 & . & 17.65 & 28.24 & 35.3 & 53 & 14.12 & 7.06 & 24.71 & & 1.71 \\
\hline & & 70 & 6 & & $\mathrm{~J}$ & & 6 & & & & 06 & 59 & 66 & & 7.28 \\
\hline & & 7.06 & 3.53 & & & & 1 & & 83 & & 10.59 & 10.59 & 4.71 & & 3.06 \\
\hline 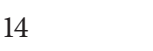 & & 0 & 0 & & & & 31.77 & & 12 & 10.59 & 28.24 & 0 & 0 & & 2.22 \\
\hline - & & 18 & 0.59 & & 28.24 & 53 & 31.77 & 63.54 & 28.24 & 21.18 & 0 & 0 & 0 & & 3.33 \\
\hline 16 & & 91.78 & 38.83 & & & 24 & 38.83 & . & 0 & . & 0 & 17.65 & 10.59 & 34 & 3.61 \\
\hline 17 & & .5 & 7.06 & & & 66 & 0 & 7.06 & 0 & & 7.06 & 35.3 & 7.06 & 61 & 1.71 \\
\hline 18 & & 7.06 & 7.06 & 10.59 & 45.89 & 63.54 & 10.59 & 31.77 & 0 & 10.59 & 35.3 & 24.71 & 28.24 & 275.34 & 3.61 \\
\hline 19 & olani & 0 & 0 & 31.77 & 31.77 & 35.3 & 24.71 & 17.65 & 24.71 & 10.59 & 21.18 & 49.42 & 31.77 & 278.87 & 3.66 \\
\hline 20 & & 14.12 & 28.24 & 28.24 & 35.3 & 17.65 & 60.01 & 35.3 & 38.83 & 0 & 10.59 & 28.24 & 45.89 & 342.41 & 4.49 \\
\hline 21 & & & 38.83 & 24.71 & 7.06 & 14.12 & 28.24 & 10.59 & 21.18 & 24.71 & 10.59 & 14.12 & 7.06 & 236.51 & 3.1 \\
\hline 22 & Penicillium spp. & 81.19 & 67.07 & 49.42 & 0 & 10.59 & 10.59 & 21.18 & 10.59 & 7.06 & 7.06 & 17.65 & 42.36 & 324.76 & 4.26 \\
\hline 23 & P. versicolor & 10.59 & 14.12 & 3.53 & 10.59 & 0 & 3.53 & 10.59 & 3.53 & 28.24 & 14.12 & 28.24 & 31.77 & 158.85 & 2.08 \\
\hline 24 & P. citrinum & 28.24 & 35.3 & 17.65 & 21.18 & 14.12 & 42.36 & 35.3 & 24.71 & 35.3 & 14.12 & 7.06 & 14.12 & 289.46 & 3.8 \\
\hline 25 & Scopulariopsis sp. & 7.06 & 14.12 & 0 & 0 & 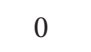 & 0 & 14.12 & 7.06 & 3.53 & 7.06 & 14.12 & 0 & 67.07 & 0.88 \\
\hline 26 & Trichoderma sp. & 28.24 & 45.89 & 35.3 & 21.18 & 28.24 & 35.3 & 56.48 & 10.59 & 21.18 & 7.06 & 14.12 & 28.24 & 331.82 & 4.35 \\
\hline \multirow[t]{2}{*}{27} & & 38.83 & 24.71 & 24.71 & 7.06 & 3.53 & 28.24 & 14.12 & 38.83 & 14.12 & 10.59 & 14.12 & 10.59 & 229.45 & 3.01 \\
\hline & Total & 716.59 & 684.82 & 790.72 & 571.86 & 610.69 & 783.66 & 649.52 & 596.57 & 469.49 & 589.51 & 571.86 & 575.39 & 7610.68 & 100 \\
\hline
\end{tabular}

significant in CFU for both groups when subjected to the same conditions for the entire year (Table 6). Analysis of the two-way ANOVA for CFU and season of the cowshed showed no significant difference in CFU's growth between indoor and outdoor cowshed colonies over the different months (Table 7).

\section{Discussion}

This study revealed that a great variety of fungal spores constitute the airborne fungal spores of the cowshed at Hesaraghatta village, Bangalore. During the survey period, indoor (7808.36 CFU/m3) and outdoor $\left(7610.68 \mathrm{CFU} / \mathrm{m}^{3}\right)$ were counted from cowshed environment. More fungal growth was observed at the cowshed. They were Acremonium sp., Alternaria alternata, Ascomycetes sp., Aspergillus sp., A. flavus, A. niger, A. oryzae, A. ochraceus, A. fumigatus, A. terreus, Curvularia sp., C. lunata, Cladosporium sp., C. cladosporioides, C. herbarum, C. acremonium, Fusarium sp., F. oxysporum, F. moniliforme, F. solani, Mucor sp., Neurospora sp., Rhizopus sp., Penicillium sp., P. versicolor, P. citrinum, P. nigricans, Scopulariopsis sp., and Trichoderma sp., were recorded Similar airborne and closely related fungal assemblages have been recorded [20].

The predominant airborne fungi were Cladosporium sp., Aspergillus sp., Aspergillus niger, and Alternaria alternata. Most cowshed investigations have reported Alternaria alternata, Aspergillus, and Cladosporium as the predominant fungal species. Sing [21] reported indoor air fungal flora of a cowshed using Andersen air sampler, out of $8448.3 \mathrm{CFU} / \mathrm{m}^{3}$ identified as Aspergillus, Cladosporium, Curvularia, Penicillium species, and so forth, were most commonly isolated types. Adhikari et al. [12] recorded 29 airborne fungal spores from the rural cowshed and a total of 24 from the suburban area. In both places, a relatively high frequency of Cladosporium sp., Aspergillus sp., Alternaria alternata, Penicillium species, and some unidentified fungi was recorded. In addition, skin prick tests were performed by them with antigenic extracts prepared from the most culture of fungi on people who were either occupationally involved are used to visit the sheds, which revealed instances of high levels of 
TABLE 3: Seasonal variations of indoor and outdoor fungal spores $\left(\mathrm{CFU} / \mathrm{m}^{3}\right)$ recorded from January 2011 to December 2011 of cowshed.

\begin{tabular}{|c|c|c|c|c|c|c|c|}
\hline \multirow{2}{*}{ Sl. number } & \multirow{2}{*}{ Genera and species } & \multicolumn{3}{|c|}{ Indoor } & \multicolumn{3}{|c|}{ Outdoor } \\
\hline & & Summer & Rainy & Winter & Summer & Rainy & Winter \\
\hline 1 & Acremonium sp. & 63.54 & 95.31 & 3.53 & 112.96 & 197.68 & 0 \\
\hline 2 & Alternaria alternata & 116.49 & 120.02 & 194.15 & 296.52 & 271.81 & 257.69 \\
\hline 3 & Ascomycetes sp. & 21.18 & 45.89 & 0 & 144.73 & 176.5 & 0 \\
\hline 4 & Aspergillus spp. & 137.67 & 144.73 & 218.86 & 250.63 & 180.03 & 384.77 \\
\hline 5 & A. flavus & 218.86 & 88.25 & 155.32 & 109.43 & 0 & 91.78 \\
\hline 6 & A. niger & 180.03 & 120.02 & 176.5 & 35.3 & 74.13 & 0 \\
\hline 7 & A. oryzae & 127.08 & 109.43 & 91.78 & 52.95 & 45.89 & 120.02 \\
\hline 8 & A. ochraceus & 17.65 & 17.65 & 14.12 & 0 & 0 & 0 \\
\hline 9 & A. fumigatus & 35.3 & 63.54 & 0 & 91.78 & 95.31 & 84.72 \\
\hline 10 & A. terreus & 31.77 & 81.19 & 109.43 & 45.89 & 3.53 & 24.71 \\
\hline 11 & Curvularia spp. & 42.36 & 38.83 & 137.67 & 45.89 & 95.31 & 38.83 \\
\hline 12 & C. lunata & 24.71 & 70.6 & 74.13 & 17.65 & 81.19 & 31.77 \\
\hline 13 & Cladosporium spp. & 317.7 & 141.2 & 176.5 & 261.22 & 63.54 & 229.45 \\
\hline 14 & C. cladosporioides & 112.96 & 141.2 & 141.2 & 102.37 & 84.72 & 45.89 \\
\hline 15 & C. herbarum & 155.32 & 102.37 & 0 & 109.43 & 60.01 & 0 \\
\hline 16 & C. acremonium & 35.3 & 63.54 & 35.3 & 98.84 & 112.96 & 42.36 \\
\hline 17 & Fusarium spp. & 130.61 & 88.25 & 120.02 & 116.49 & 0 & 158.85 \\
\hline 18 & F. oxysporum & 77.66 & 45.89 & 84.72 & 28.24 & 42.36 & 60.01 \\
\hline 19 & F. moniliforme & 155.32 & 28.24 & 88.25 & 130.61 & 77.66 & 67.07 \\
\hline 20 & F. solani & 70.6 & 116.49 & 81.19 & 123.55 & 74.13 & 81.19 \\
\hline 21 & Mucor sp. & 56.48 & 45.89 & 105.9 & 141.2 & 84.72 & 116.49 \\
\hline 22 & Neurospora sp. & 31.77 & 24.71 & 0 & 0 & 0 & 0 \\
\hline 23 & Rhizopus sp. & 105.9 & 105.9 & 105.9 & 74.13 & 67.07 & 95.31 \\
\hline 24 & Penicillium spp. & 120.02 & 88.25 & 127.08 & 70.6 & 45.89 & 208.27 \\
\hline 25 & P. versicolor & 63.54 & 88.25 & 105.9 & 17.65 & 56.48 & 84.72 \\
\hline 26 & P. citrinum & 102.37 & 63.54 & 84.72 & 95.31 & 109.43 & 84.72 \\
\hline 27 & P. nigricans & 67.07 & 112.96 & 3.53 & 0 & 0 & 0 \\
\hline 28 & Scopulariopsis sp. & 0 & 21.18 & 10.59 & 0 & 31.77 & 35.3 \\
\hline 29 & Trichoderma sp. & 70.6 & 67.07 & 112.96 & 120.02 & 95.31 & 116.49 \\
\hline 30 & Unidentified & 52.95 & 81.19 & 84.72 & 63.54 & 77.66 & 88.25 \\
\hline & Total & 2742.81 & 2421.58 & 2643.97 & 2756.93 & 2305.09 & 2548.66 \\
\hline
\end{tabular}

TABLE 4: Comparison between indoor and outdoor air of cowshed.

\begin{tabular}{lcc}
\hline Variables & Indoor & Outdoor \\
\hline Concentration & $6.51 \pm 0.94$ & $6.34 \pm 0.95$ \\
Temperature & $23.27 \pm 1.99$ & $23.27 \pm 1.99$ \\
Relative humidity & $67.32 \pm 7.28$ & $67.32 \pm 7.28$ \\
Wind speed & $5.43 \pm 1.60$ & $5.43 \pm 1.60$ \\
Rain fall & $67.04 \pm 73.71$ & $67.04 \pm 73.71$ \\
\hline
\end{tabular}

allergenicity. Adhikari et al. [22] recorded 31 fungal spore types and 35 types of viable CFUs using Andersen two-stage viable sampler.

Factors such as building dampness, indoor temperature, relative humidity, and hygiene conditions indoors and in the surrounding environment favour the growth and proliferation of fungi including the pathogenic species [23]. There is clinical evidence that exposure to mould and other dampness-related microbial agents increases the risk of rare
TABLE 5: Pearson's correlation between meteorological variables of cowshed.

\begin{tabular}{lccccc}
\hline Linear correlation table & Conc. & $T$ & RH & WS & RF \\
\hline Concentration & 1.000 & & & & \\
Temperature & 0.053 & 1.000 & & & \\
Relative humidity & -0.503 & -0.408 & 1.000 & & \\
Wind speed & 0.129 & 0.141 & 0.268 & 1.000 & \\
Rain fall & -0.249 & 0.345 & 0.191 & 0.095 & 1.000 \\
\hline
\end{tabular}

conditions such as hypersensitivity, pneumonitis, allergic alveolitis, chronic rhinosinusitis, and allergic fungal sinusitis [24]. This could be because of improper management of the indoor environment and poor ventilation. In the naturally continuous mixing of indoor and outdoor air, the concentration of fungi can be two to five times higher than the outdoor level [25]. However, the spore concentration has been observed to be much lower in outdoor (control) environment. Because many people spend as much as $90 \%$ 


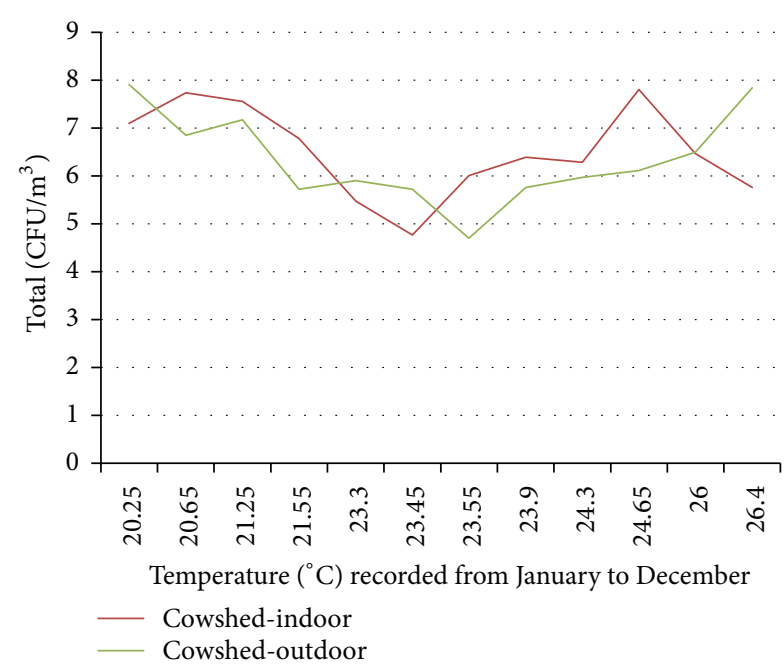

(a)

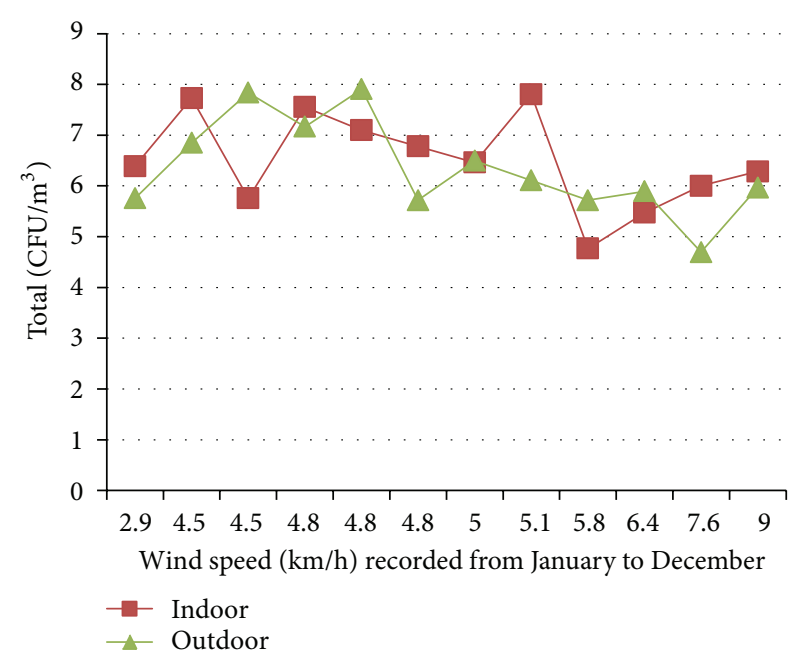

(c)

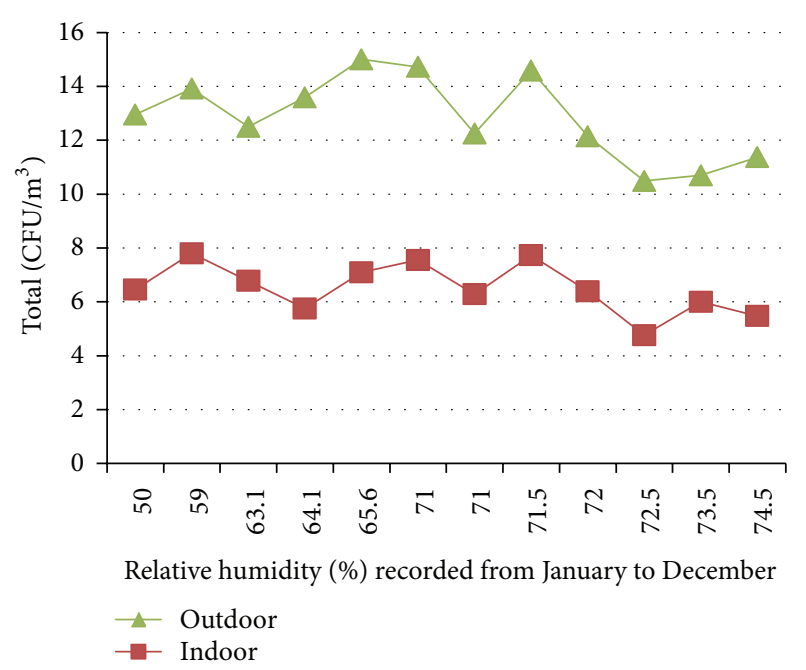

(b)

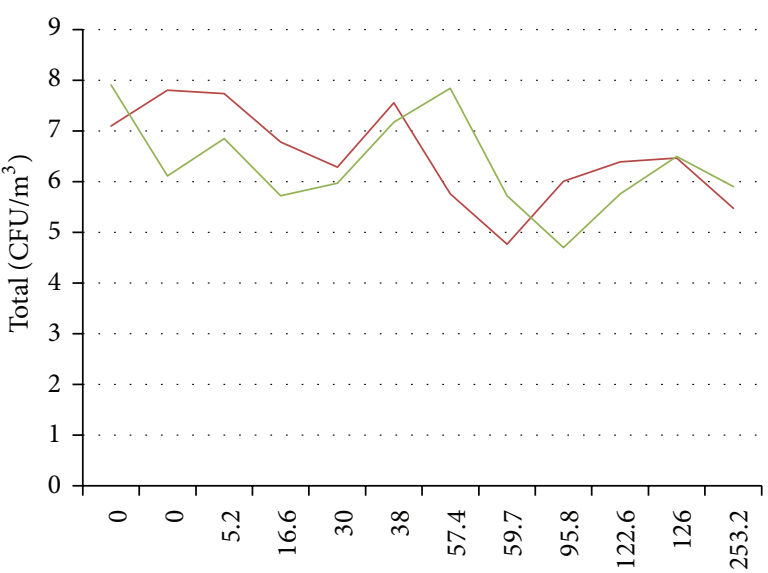

Rain fall (mm) recorded from January to December

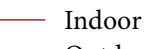

_ Outdoor

FIGURE 1: Distribution of the fungal spores $\left(\mathrm{CFU} / \mathrm{m}^{3}\right)$ and meteorological parameters recorded.

TABLE 6: One-way ANOVA for CFU of cowshed.

\begin{tabular}{lccccc}
\hline $\begin{array}{l}\text { One-way } \\
\text { ANOVA } \\
\text { table }\end{array}$ & $\begin{array}{c}\text { Sum of } \\
\text { squares }\end{array}$ & $\begin{array}{c}\text { Degrees of } \\
\text { freedom }\end{array}$ & $\begin{array}{c}\text { Mean } \\
\text { squares }\end{array}$ & $F$-ratio & $P$ value \\
\hline $\begin{array}{l}\text { Between } \\
\text { variation }\end{array}$ & 0.16 & 1 & 0.16 & 0.18 & 0.67 \\
$\begin{array}{l}\text { Within } \\
\text { variation }\end{array}$ & 19.55 & 22 & 0.88 & & \\
\hline $\begin{array}{l}\text { Total } \\
\text { variation }\end{array}$ & 19.72 & 23 & & & \\
\hline
\end{tabular}

of their time indoors, the health risk of indoor air pollutants is of critical public health concern. Studies have shown association between reported indoor dampness and health outcomes, including respiratory symptoms, headache, and upper respiratory airway infections [26].

The total spore concentration and CFU in cowshed were observed during various seasons, which revealed that indoor and outdoor higher total spore and CFU concentration levels were maximum in summer season followed by winter season and then rainy season. The possible reason could be due to the vigorous growth of plant in summer simultaneously allowing the growth of airborne fungi along with increased temperature and therefore favoring fungal growth [27]. The winter season in India belongs to the months between November and February, summer between March and June, and rainy between July and October. The $\mathrm{CFU} / \mathrm{m}^{3}$ growth for cowshed was the lowest during the rainy season when the temperature was around $23^{\circ} \mathrm{C}$. The reason could be due to the washing of fungal air spores from the atmosphere due to precipitation. Immediately after rainy seasons, the $\mathrm{CFU} / \mathrm{m}^{3}$ 
TABLE 7: Two-way ANOVA for CFU and season of the cowshed.

\begin{tabular}{lccccc}
\hline $\begin{array}{l}\text { Two-way } \\
\text { ANOVA } \\
\text { table }\end{array}$ & $\begin{array}{c}\text { Sum of } \\
\text { squares }\end{array}$ & $\begin{array}{c}\text { Degrees of } \\
\text { freedom }\end{array}$ & $\begin{array}{c}\text { Mean } \\
\text { squares }\end{array}$ & $F$-ratio & $P$ value \\
\hline Colony & 0.04 & 1 & 0.04 & 2.65 & 0.24 \\
Season & 0.94 & 2 & 0.47 & 30.83 & 0.03 \\
Error & 0.03 & 2 & 0.01 & & \\
\hline Total & 1.01 & 5 & & & \\
\hline
\end{tabular}

increases since there was an increase in temperature and abundance in dead plant material favoring the spore relation conditions for fungi, and ventilation was also inadequate there. Such observations have been recorded by investigation carried out by Aydogdu and Asan [28].

The differences in cowshed were observed with respect to the CFU's and spore concentrations of individual fungal types with average monthly concentrations, but no significant difference was observed when the total overall yearly concentrations were considered. The possible reason might be the insignificant changes of environmental factors as well as the fungal growth substrates at the cowshed during the oneyear sampling. It is an established fact that temperature and relative humidity are two important factors for fungal spore generation, release, and dispersal, particularly in indoor environments [29, 30]. Higher temperature and relative humidity in the months of rainy season and associated higher concentration of culturable fungal spores in the cowshed are supporting the observations of earlier researchers. The dominance of Aspergillus, Penicillium, and Cladosporium in all monitored locations was in agreement with Shelton et al. [31], who reported that these were the dominant culturable airborne fungi in indoor buildings in United States and elsewhere. There were also reports that Aspergillus can be used as an indicator for fungal contamination in buildings [32]. According to Nielsen [33], Aspergillus, Penicillium, Fusarium, Alternaria, and Trichoderma are the most frequently found fungal genera indoor and some species of these genera are main mycotoxin producers that can cause mycotoxicoses. This toxic response sometime leads to deterioration of liver or kidney functions if ingested by humans through food [34].

A series of fungal treatments and other remediation steps were carried out to improve conditions that were favorable for the establishment and growth of the fungi. During the treatment, special clothing with rubber gloves and mask was used to protect the skin and eyes. Besides, high efficiency particulate air respirators were used during the fungal treatment. The effectiveness of the treatment was proven by the decreasing numbers of CFU during air sampling. Although most fungi have periods of the year when their spores are more prevalent than others, some can be found virtually all year round. Concentration of fungal spores in outdoor environments is usually high, especially during the rainy season. Although many spore types are washed from the atmosphere during rainfall, other spore types only occur in rainy weather. The air is abundant of fungal spores although it is not a good medium for growth unlike the soil, water, surfaces of living organisms, and nonliving materials.

\section{Conclusion}

This study was carried out in the animal rearing houses; it clearly revealed the concentration of different fungal species in the environment. The data of fungal spore content in indoor and outdoor environment helped us to prepare the fungal spore calendar on this region. The seasonal periodicity information of the major allergenic spores will be valuable for susceptible patients to take respiratory protection measures during the peak time. The fungal spore calendar and prediction model will be helpful to forecast the allergenic fungal spore load in the air of Hesaraghatta village. Respiratory allergic problems and hospital admission with relevant diseases of that zone are related to the presence of airborne allergenic fungal spores.

\section{Conflict of Interests}

The authors declare that there is no conflict of interests regarding the publication of this paper.

\section{Acknowledgments}

This study was supported by the Department of Microbiology and Biotechnology, Bangalore University, Bangalore. The authors are grateful to University Grants CommissionBasic Science Research Fellowship, New Delhi, for financial support of this research. The authors appreciate the cowshed workers for providing invaluable support and technical assistance during the sampling time.

\section{References}

[1] L. S. Ruzer and N. H. Harley, Aerosols Handbook: Measurement, Dosimetry and Health Effects, CRC Press, Florida, Fla, USA, 2005.

[2] B. Lighthart, "Mini-review of the concentration variations found in the alfresco atmospheric bacterial populations," Aerobiologia, vol. 16, no. 1, pp. 7-16, 2000.

[3] H. A. Burge, "Fungi: toxic killers or unavoidable nuisances?" Annals of Allergy, Asthma and Immunology, vol. 87, no. 6, pp. 52-56, 2001.

[4] R. K. Bush and J. M. Portnoy, "The role and abatement of fungal allergens in allergic diseases," Journal of Allergy and Clinical Immunology, vol. 107, no. 3, pp. S430-S440, 2001.

[5] W. E. Horner, A. Helbling, J. E. Salvaggio, and S. B. Lehrer, "Fungal allergens," Clinical Microbiology Reviews, vol. 8, no. 2, pp. 161-179, 1995.

[6] Z. Cvetnić and S. Pepeljnjak, "Distribution and mycotoxinproducing ability of some fungal isolates from the air," Atmospheric Environment, vol. 31, no. 3, pp. 491-495, 1997.

[7] P. N. Black, A. A. Udy, and S. M. Brodie, "Sensitivity to fungal allergens is a risk factor for life-threatening asthma," Allergy, vol. 55, no. 5, pp. 501-504, 2000.

[8] D. C. Christiani, A. Velazquez, M. Wilcox, and S. A. Olenchock, "Airborne endotoxin concentrations in various work 
areas within a cotton mill in Central America," Environmental Research, vol. 60, no. 2, pp. 187-192, 1993.

[9] B. Ostro, M. Lipsett, J. Mann, H. Braxton-Owens, and M. White, "Air pollution and exacerbation of asthma in African-American children in Los Angeles," Epidemiology, vol. 12, no. 2, pp. 200208, 2001.

[10] X. Zhao and Z. Yang, "Explore the relation to air fungi concentration between office buildings and sick buildings," Modern Medicine and Public Health, vol. 23, pp. 1409-1411, 2007.

[11] A. Meriggi, S. Ricci, M. Bruni, and R. Corsico, "Aerobiological monitoring for fungal spores in a rehabilitation hospital in Northern Italy," Aerobiologia, vol. 12, no. 4, pp. 233-237, 1996.

[12] A. Adhikari, M. M. Sen, S. Gupta-Bhattacharya, and S. Chanda, "Studies on airborne fungal spores from two indoor cowsheds of suburban and rural areas of West Bengal, India," Indoor and Built Environment, vol. 8, no. 4, pp. 221-229, 1999.

[13] H. Ajoudanifar, M. T. Hedayati, S. Mayahi, A. Khosravi, and B. Mousavi, "Volumetric assessment of airborne indoor and outdoor fungi at poultry and cattle houses in the Mazandaran Province, Iran," Arhiv za Higijenu Rada i Toksikologiju, vol. 62, no. 3, pp. 243-248, 2011.

[14] M. Sowiak, K. Bródka, A. Buczyńska et al., "An assessment of potential exposure to bioaerosols among swine farm workers with particular reference to airborne microorganisms in the respirable fraction under various breeding conditions," Aerobiologia, vol. 28, no. 2, pp. 121-133, 2012.

[15] C. P. Newhouse and E. Levetin, "Correlation of environmental factors with asthma and rhinitis symptoms in Tulsa, OK," Annals of Allergy, Asthma \& Immunology, vol. 92, no. 3, pp. 356366, 2004.

[16] S. S. Okten, A. Asan, Y. Tungan, and M. Ture, "Airborne fungal concentrations in East Patch of Edrine City (Turkey) in autumn using two sampling methods," Trakya University Journal of Science, vol. 6, no. 1, pp. 97-106, 2005.

[17] J. Bartra Tomas, "Mesa redonda: estado actual de la alergia a hongos," Alergologiae Inmunologia Clinica, vol. 18, no. 3, pp. 106-121, 2003.

[18] F. J. Rodríguez-Rajo, A. Dopazo, and V. Jato, “Environmental factors affecting the start of pollen season and concentrations of airborne Alnus pollen in two localities of Galicia (NW Spain)," Annals of Agriculture and Environmental Medicine, vol. 11, no. 1, pp. 35-44, 2004.

[19] A. A. Andersen, "New sampler for collection, sizing and enumeration of viable airborne particles," Journal of Bacteriology, vol. 76, pp. 471-484, 1958.

[20] L. Curtis, M. Ross, V. Persky et al., "Bioaerosol concentrations in the Quad Cities 1 year after the 1993 Mississippi River floods," Indoor and Built Environment, vol. 9, no. 1, pp. 35-43, 2000.

[21] A. B. Sing, "An overview of agricultural asthma in farm workers," Indian Journal of Aerobiology and Applications Immunology, vol. 12, pp. 71-74, 1998.

[22] A. Adhikari, M. M. Sen, S. Gupta-Bhattacharya, and S. Chanda, "Volumetric assessment of airborne fungi in two sections of a rural indoor dairy cattle shed," Environment International, vol. 29, no. 8, pp. 1071-1078, 2004.

[23] C. G. Bornehag, "Dampness in buildings and health nordic interdisciplinary review of the scientific evidence on associations between exposure to "dampness" in buildings and health effects (NORDDAMP)," Indoor Air, vol. 11, no. 2, pp. 72-86, 2001.
[24] WHO, WHO Guidelines for Indoor Air Quality: Dampness and Mould, Edited by E. Heseltine and J. Rosen, World Health Organization, 2009.

[25] US EPA Green Building Workgroup, "Buildings and the environment: a statistical summary," US EPA Report, 2004.

[26] A. P. Verhoeff and H. A. Burge, "Health risk assessment of fungi in home environments," Annals of Allergy, Asthma and Immunology, vol. 78, no. 6, pp. 544-556, 1997.

[27] Z. G. Fang, Z. Y. Ouyang, L. F. Hu, X. K. Wang, H. Zheng, and X. Q. Lin, "Culturable airborne fungi in outdoor environments in Beijing, China," Science of the Total Environment, vol. 350, no. 1-3, pp. 47-58, 2005.

[28] H. Aydogdu and A. Asan, "Airborne fungi in child day care centers in Edirne City, Turkey," Environmental Monitoring and Assessment, vol. 147, no. 1-3, pp. 423-444, 2008.

[29] B. Herrero and P. Zaldivar, "Effects of meteorological factors on the levels of Alternaria and Cladosporium spores in the atmosphere of Palencia, 1990-92," Grana, vol. 36, no. 3, pp. 180184, 1997.

[30] T. O. Larsen and J. C. Frisvad, "Production of volatiles and mycotoxins in conidia of common Penicillium and Aspergillus," in Health Implications of Fungi in Indoor Environments, R. A. Samson and B. Flannigan, Eds., pp. 251-279, Elsevier, Amsterdam, The Netherlands, 1994.

[31] B. G. Shelton, K. H. Kirkland, W. D. Flanders, and G. K. Morris, "Profiles of airborne fungi in buildings and outdoor environments in the United States," Applied and Environmental Microbiology, vol. 68, no. 4, pp. 1743-1753, 2002.

[32] J. B. Hicks, E. T. Lu, R. de Guzman, and M. Weingart, "Fungal types and concentrations from settled dust in normal residences," Journal of Occupational and Environmental Hygiene, vol. 2, no. 10, pp. 481-492, 2005.

[33] K. F. Nielsen, "Mycotoxin production by indoor molds," Fungal Genetics and Biology, vol. 39, no. 2, pp. 103-117, 2003.

[34] W. F. O. Marasas, J. D. Miller, R. T. Riley, and A. Viscontini, "Fusmonisins occurrence, toxicology, metabolism and risk assessment," in Fursarium : Paul E. Nelson, Memorial Symposium, B. A. Summerell, J. F. Leslie, D. Backhouse, and W. L. Bryden, Eds., pp. 332-359, APS Press, Saint Paul, Minn, USA, 2011. 

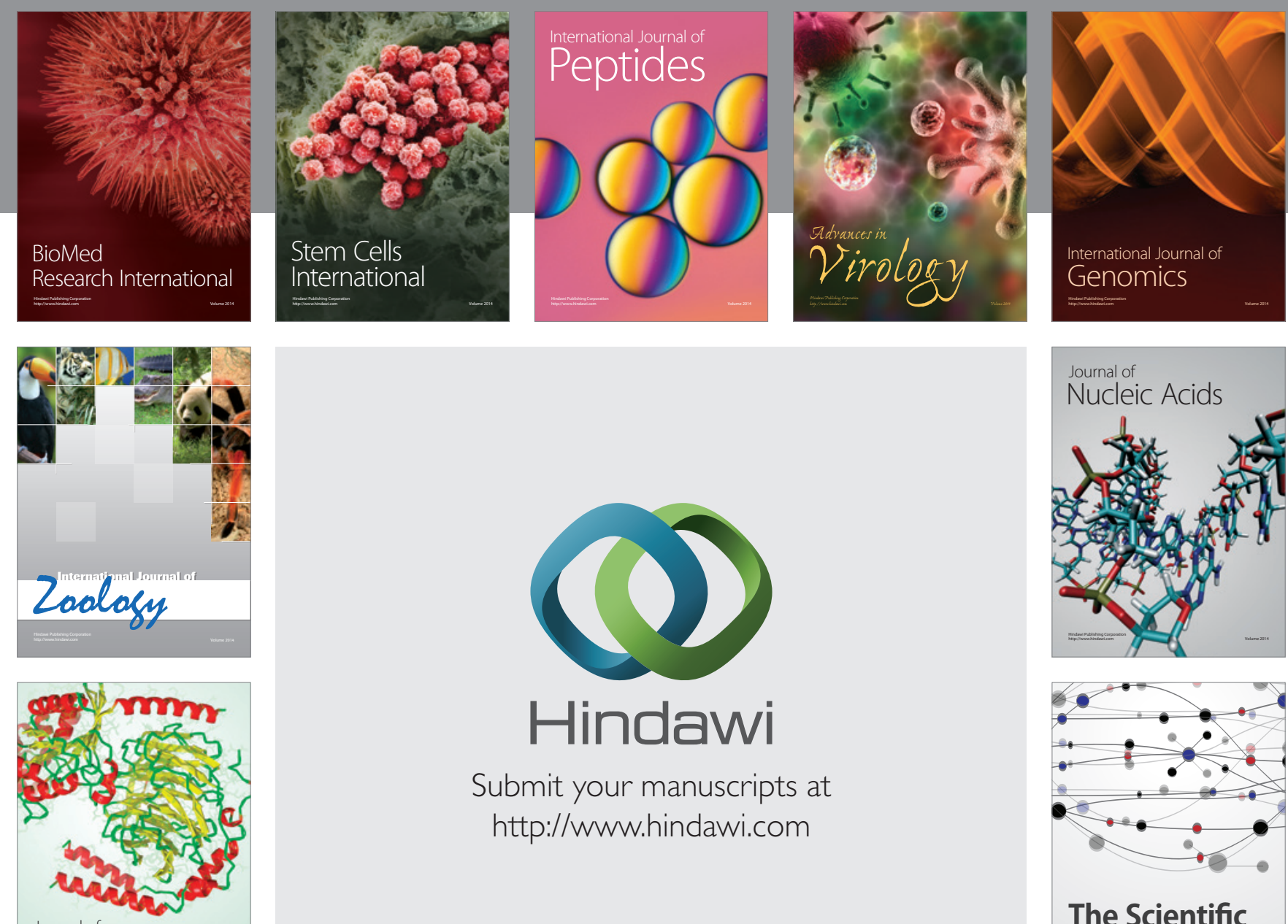

Submit your manuscripts at

http://www.hindawi.com

Journal of
Signal Transduction
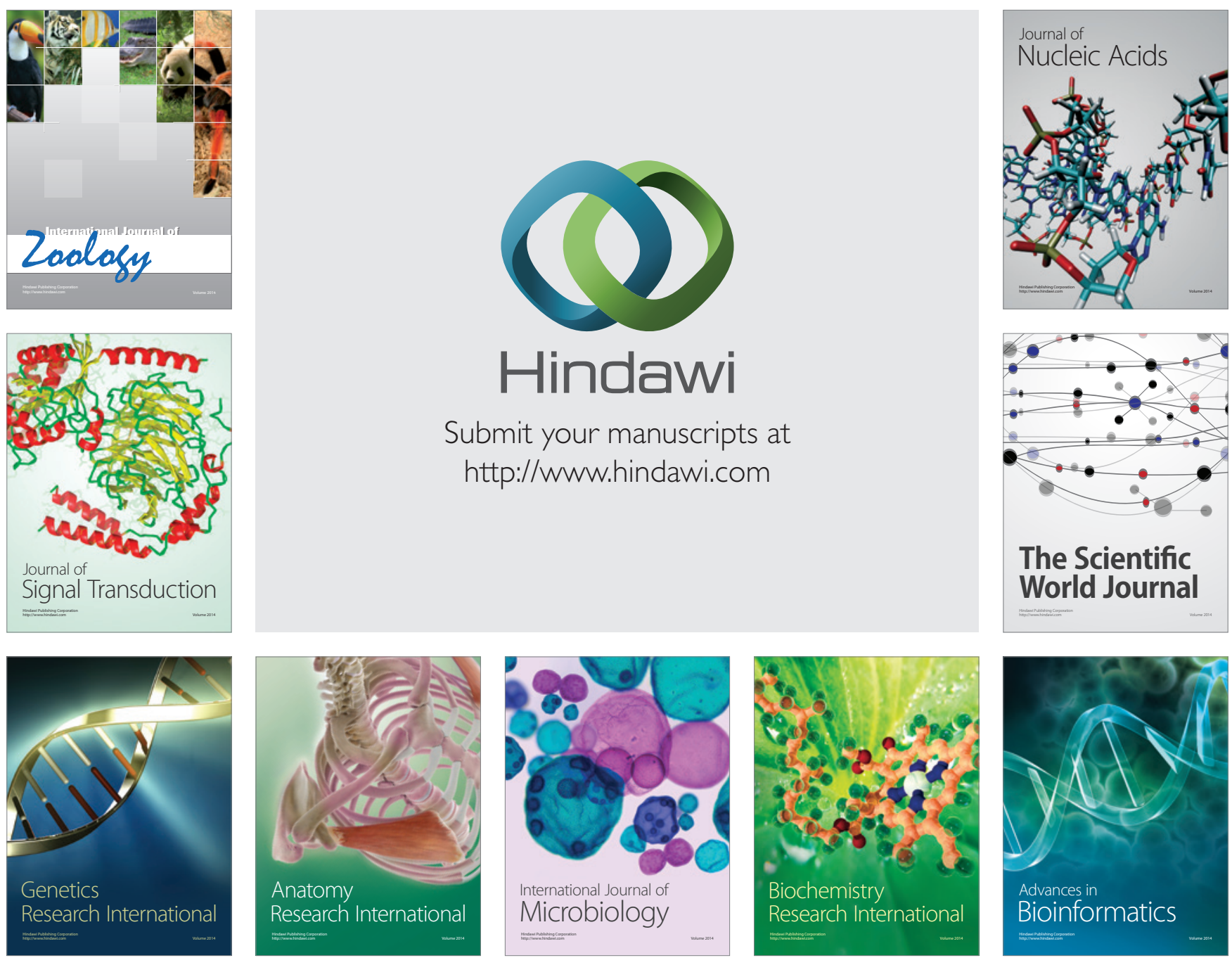

The Scientific World Journal
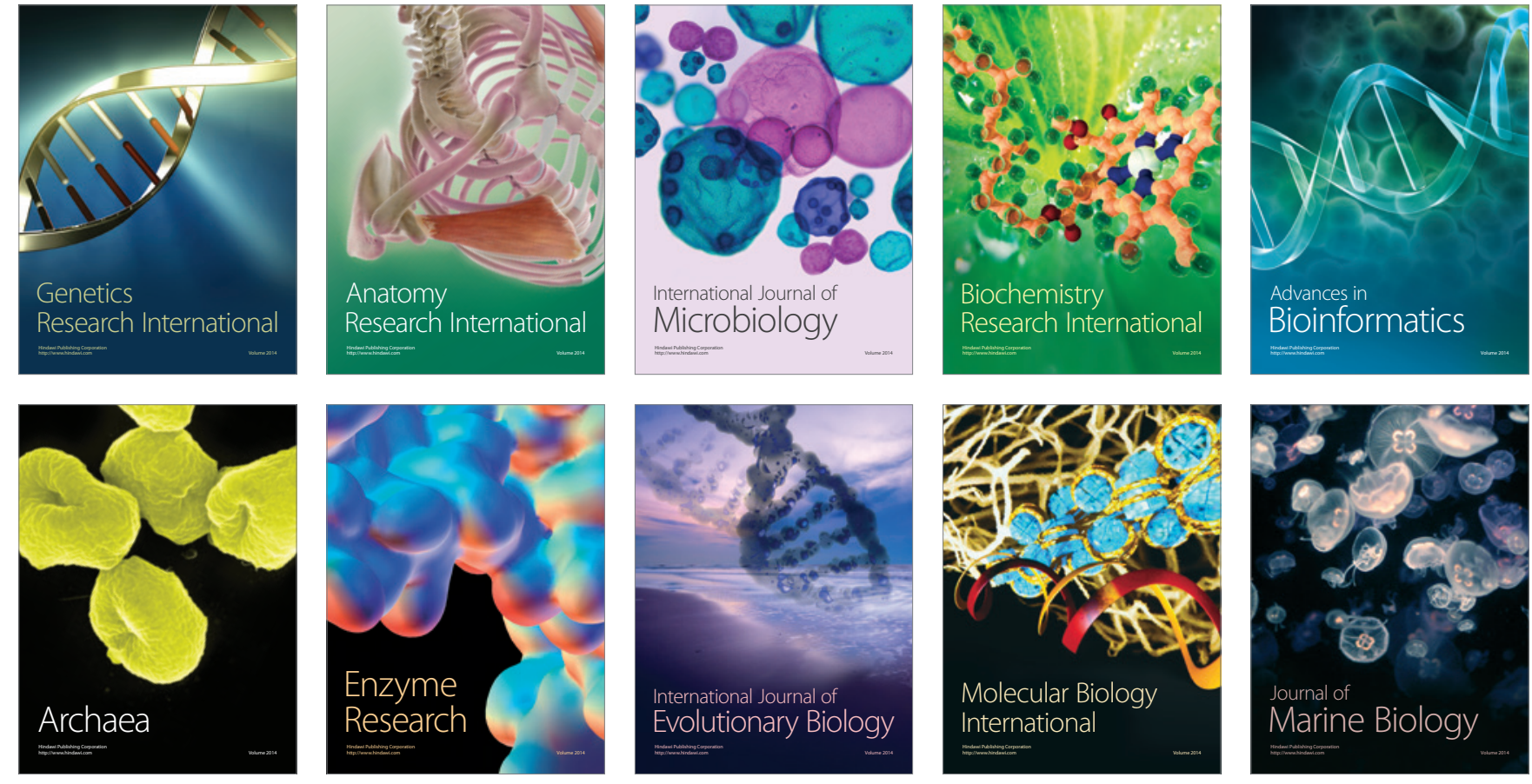